\title{
Worse long-term survival after off-pump than on-pump coronary artery bypass grafting
}

\author{
Hisato Takagi, MD, PhD, and Takuya Umemoto, $\mathrm{MD}, \mathrm{PhD}$, for the All-Literature Investigation of \\ Cardiovascular Evidence (ALICE) Group
}

\begin{abstract}
Objective: To determine whether off-pump coronary artery bypass grafting (CABG) is associated with worse long-term survival compared with on-pump CABG. We performed a meta-analysis of adjusted observational studies and randomized controlled trials.
\end{abstract}

\begin{abstract}
Methods: MEDLINE, EMBASE, and the Cochrane Central Register of Controlled Trials were searched through March 2014. Eligible studies were randomized controlled trials and adjusted observational studies (in which appropriate statistical methods adjusting for confounders had been used) of off-pump versus on-pump CABG that had reported long-term ( $\geq 5$-year) all-cause mortality as an outcome.
\end{abstract}

Results: Of 478 potentially relevant studies screened initially, 5 randomized trials and 17 observational studies, enrolling a total of 104,306 patients, were identified and included. A pooled analysis of all 22 studies demonstrated a statistically significant $7 \%$ increase in long-term all-cause mortality with off-pump relative to on-pump CABG (hazard ratio, 1.07; 95\% confidence interval, $1.03-1.11 ; P=.0003$ ). Although a pooled analysis of 5 randomized trials (1486 patients) demonstrated a statistically nonsignificant $14 \%$ increase in mortality with off-pump relative to on-pump CABG (hazard ratio, 1.14; 95\% confidence interval, 0.84-1.56; $P=.39$ ), another pooled analysis of 17 observational studies (102,820 patients) demonstrated a statistically significant $7 \%$ increase in mortality with off-pump relative to on-pump CABG (hazard ratio, $1.07 ; 95 \%$ confidence interval, $1.03-1.11 ; P=.0004)$.

Conclusions: A meta-analysis of 22 studies, enrolling a total of $>100,000$ patients, showed that off-pump CABG is likely associated with worse long-term ( $\geq 5$-year) survival compared with on-pump CABG. (J Thorac Cardiovasc Surg 2014;148:1820-9)

See related commentary on pages 1829-31.

Supplemental material is available online.

A Cochrane systematic review ${ }^{1}$ published in 2012 of 30 randomized clinical trials did not demonstrate any significant benefit for off-pump compared with on-pump coronary artery bypass grafting (CABG) regarding mortality, stroke, or myocardial infarction. In contrast, Møller and colleagues ${ }^{1}$ observed, in a pooled analysis of 17 trials, ${ }^{2-18}$ better late (>30-day) survival in the group of patients

From the Department of Cardiovascular Surgery, Shizuoka Medical Center, Shizuoka, Japan.

Disclosures: Authors have nothing to disclose with regard to commercial support. Received for publication April 9, 2014; revisions received May 6, 2014; accepted for publication May 16, 2014; available ahead of print June 17, 2014.

Address for reprints: Hisato Takagi, MD, PhD, Department of Cardiovascular Surgery, Shizuoka Medical Center, 762-1 Nagasawa, Shimizu-cho, Sunto-gun, Shizuoka 411-8611, Japan (E-mail: kfgth973@ybb.ne.jp).

$0022-5223 / \$ 36.00$

Copyright (c) 2014 by The American Association for Thoracic Surgery

http://dx.doi.org/10.1016/j.jtcvs.2014.05.034 undergoing on-pump CABG with cardiopulmonary bypass and cardioplegic arrest. The follow-up duration of the included trials, however, was $<3$ years in 12 trials, ${ }^{3-6,8-14,17} \geq 3$ but $<5$ years in 3 trials, ${ }^{7,16,18}$ and $\geq 5$ years in only 2 trials. ${ }^{2,15}$ The results of our previous 2012 meta-analysis ${ }^{19}$ of 14 randomized controlled trials $2,4,6,8,9,14,15,17,18,20-24$ suggested that off-pump CABG might increase late ( $\geq 1$-year) all-cause mortality by $35 \%$ compared with on-pump CABG. The follow-up duration of the included trials was $<3$ years in 8 trials, $4,6,8,9,14,17,22,23$ $\geq 3$ but $<5$ years in 1 trial, $^{18}$ and $\geq 5$ years in 5 trials. ${ }^{2,15,20,21,24}$ To determine whether off-pump CABG is associated with worse long-term ( $\geq 5$-year) survival compared with on-pump CABG, we performed a metaanalysis of adjusted observational studies (in which appropriate statistical methods adjusting for confounders had been used) and randomized controlled trials.

\section{METHODS}

\section{Search Strategy}

All prospective randomized controlled trials and adjusted observational comparative studies of off-pump versus on-pump CABG that had reported long-term ( $\geq 5$-year) all-cause mortality were identified using a 2-level search strategy. First, databases, including MEDLINE, EMBASE, and the Cochrane Central Register of Controlled Trials, were searched through March 2014 using Web-based search engines (PubMed and OVID). 


$$
\begin{aligned}
& \text { Abbreviations and Acronyms } \\
& \begin{aligned}
\text { CABG } & =\text { coronary artery bypass grafting } \\
\text { CI } & =\text { confidence interval } \\
\text { HR } & =\text { hazard ratio } \\
\text { PS } & =\text { propensity score } \\
\text { RR } & =\text { risk ratio }
\end{aligned}
\end{aligned}
$$

Second, relevant studies were identified through a manual search of secondary sources, including the references of the initially identified studies, and a search of reviews and commentaries. All references were downloaded for consolidation, elimination of duplicates, and additional analysis. The text keywords included "off-pump"; "long-term" or "late"; and "mortality," "death," "deaths," "survival," "outcome," "outcomes," or "follow-up."

\section{Study Selection and Data Abstraction}

Studies considered for inclusion had to meet the following criteria: the design was a prospective randomized controlled clinical trial or prospective or retrospective adjusted observational comparative study (in which appropriate statistical methods adjusting for confounders had been used); the study population was patients undergoing $\mathrm{CABG}$; the patients had been assigned to off-pump versus on-pump CABG; and the main outcomes included long-term all-cause mortality. We defined long-term mortality as death during $\geq 5$ years of follow-up in the present analysis. When duplicate reports from the same study were identified, only the most recent publication, or the one with the longest follow-up period, was included. Data regarding detailed inclusion criteria, duration of follow-up, and mortality were abstracted (as available) from each individual study. We extracted a crude (unadjusted) hazard ratio (HR) and $95 \%$ confidence interval (CI) of off-pump versus on-pump CABG for mortality from a randomized trial and an adjusted HR (using appropriate statistical methods such as propensity-score [PS] matching, PS stratification, PS-adjusted Cox proportional hazards regression, multivariable Cox proportional hazards regression, and multivariable Poisson regression) from an observational study. When the HR was unavailable from a randomized trial or PS-matched study, the number of deaths in both the off-pump and on-pump groups was used to generate the odds ratio and $95 \% \mathrm{CI}$ instead of the $\mathrm{HR}$.

\section{Statistical Analysis}

We conducted a meta-analysis of the summary statistics from the individual studies. Study-specific estimates were combined using inverse variance-weighted averages of logarithmic HRs in both fixed-effects and random-effects models. Between-study heterogeneity was analyzed using the standard chi-square test. If no significant statistical heterogeneity was identified, the fixed-effect estimate was used preferentially as the summary measure. Sensitivity analyses were performed to assess the contribution of each study to the pooled estimate by excluding individual studies one at a time and recalculating the pooled HR estimates for the remaining studies. To assess the effect of differential adjustment methods among the observational studies on the pooled estimate, the effects of off-pump CABG on mortality were explored separately in the PS-matched studies. Publication bias was assessed graphically using a funnel plot and mathematically using an adjusted rank correlation and linear regression test. All analyses were conducted using Review Manager, version 5.2 (Nordic Cochrane Centre, Copenhagen, Denmark), and Comprehensive Meta-Analysis, version 2 (Biostat, Englewood, NJ).

\section{RESULTS}

\section{Search Results}

Of 478 potentially relevant studies screened initially, 5 prospective randomized controlled clinical trials $2,15,20,21,24$ and 17 prospective or retrospective adjusted observational

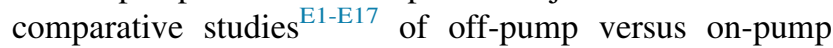
CABG reporting long-term ( $\geq 5$-year) all-cause mortality were identified and included. In total, our meta-analysis included data on 104,306 patients assigned to off-pump or on-pump CABG. The study design and patient characteristics are summarized in Table 1, details of revascularization in Table 2, and graft patency and cardiac events in Table 3. The number of grafts (or distal anastomoses) per patient was reported in 16 studies and was significantly greater for on-pump than off-pump CABG in 8 of the 16 studies (Table 2). The completeness of revascularization was provided in 8 studies, and the index of the completeness of revascularization (or the frequency of complete revascularization) was reported to be significantly greater with on-pump than with off-pump CABG in 6 of the 8 studies (Table 2). In only 1 PS-matched study by $\mathrm{Hu}$ and colleagues, ${ }^{\mathrm{E} 9}$ was repeat revascularization, angina, and rehospitalization for cardiac reasons significantly more frequent with off-pump than with on-pump CABG (Table 3).

\section{Primary Meta-Analysis}

A pooled analysis of all 22 studies (104,306 patients) demonstrated a statistically significant $7 \%$ increase in long-term ( $\geq 5$-year) all-cause mortality with off-pump relative to on-pump CABG in the fixed-effects model (HR, $1.07 ; \quad 95 \% \quad$ CI, $1.03-1.11 ; \quad P$ for overall effect $=.0003$; Figure 1$)$. Minimal trial heterogeneity ( $P$ for heterogeneity $=.09$ ) was present and, accordingly, little difference in the pooled result from random-effects modeling (HR, 1.06; 95\% CI, 1.01-1.12; $P$ for overall effect $=.03)$. Although a pooled analysis of 5 randomized controlled trials (1486 patients) demonstrated a statistically nonsignificant $14 \%$ increase in mortality with off-pump relative to on-pump CABG (fixed-effects HR, 1.14; $95 \%$ CI, $0.84-1.56 ; \quad P$ for overall effect $=.39 ; P$ for heterogeneity $=.38$ ), another pooled analysis of 17 adjusted observational studies $(102,820$ patients $)$ demonstrated a statistically significant $7 \%$ increase in mortality with off-pump relative to on-pump CABG (fixed-effects HR, $1.07 ; 95 \% \mathrm{CI}, 1.03$ to $1.11 ; P$ for overall effect $=.0004 ; P$ for heterogeneity $=.06$; Figure 1$)$.

\section{Sensitivity Analyses}

To assess the effect of qualitative heterogeneity in study design and patient selection on the pooled-effect estimate, we performed several sensitivity analyses. First, we excluded the highest weight $(43.0 \%)$ and largest size (35,644 patients) study by Bakaeen and colleagues ${ }^{\mathrm{E} 2}$ (Continuous Improvement in Cardiac Surgery Program). However, combining the remaining studies still generated statistically significant results favoring on-pump CABG (fixed-effects HR, 1.07; 95\% CI, 1.02-1.13; $P$ for overall 
effect $=.003 ; P$ for heterogeneity $=.07)$. Second, we sequentially excluded the second highest weight $(12.0 \%)$ study by Wu and colleagues ${ }^{\mathrm{E} 12}$ (New York State's Cardiac Surgery Reporting System) and the second largest size $\left(12,874\right.$ patients) study by Cooper and colleagues. ${ }^{\text {E3 }}$ Without the New York State's Cardiac Surgery Reporting
System $^{\text {E12 }}$ (fixed-effects HR, 1.06; 95\% CI, 1.02 to 1.11 ; $P$ for overall effect $=.001 ; P$ for heterogeneity $=.08$ ) or the study by Cooper and colleagues ${ }^{\mathrm{E} 3}$ (fixed-effects HR, $1.07 ; 95 \%$ CI, 1.03 to $1.11 ; P$ for overall effect $=.0006$; $P$ for heterogeneity $=.07$ ), the benefit for on-pump CABG in the pooled analysis of the remaining studies

TABLE 1. Trial design and patient characteristics

\begin{tabular}{|c|c|c|c|c|c|}
\hline \multicolumn{6}{|c|}{ Study } \\
\hline Name & Duration & Inclusion criteria & Adjustment & Follow-up & Subgroup \\
\hline \multicolumn{6}{|l|}{ Randomized controlled trial } \\
\hline $\begin{array}{l}\text { Angelini (BHACAS } \\
\quad 1 \text { and } 2 \text { ), }{ }^{20} 2009\end{array}$ & $\begin{array}{l}\text { March } 1997 \text { to August 1998, } \\
\text { BHACAS 1; September } 1998 \\
\text { to November 1999, BHACAS } 2\end{array}$ & $\begin{array}{l}\text { Recent MI }(<1 \mathrm{mo}) \text { and } \\
\text { pronounced disease of distal } \\
\text { branches of circumflex artery } \\
\text { excluded in BHACAS } 1 \text { but } \\
\text { not BHACAS } 2^{*}\end{array}$ & - & $\begin{array}{l}75.5 \pm 20.6 \text { mo, off-pump } \\
76.7 \pm 19.3 \text { mo, on-pump }\end{array}$ & BHACAS $1 *$ \\
\hline & & & & & $\begin{array}{l}\text { BHACAS } 2 * \\
\text { Total }^{*}\end{array}$ \\
\hline Karolak, ${ }^{21} 2007$ & August 1999 to March 2003 & $\begin{array}{r}\mathrm{EF} \geq 30 \% \text {; nonemergency } \\
\text { primary isolated } \mathrm{CABG}\end{array}$ & - & 5 y (mean, 3.8; IQR, 3.4-4.4) & - \\
\hline Hueb (MASS III), ${ }^{15} 2010$ & - & Primary isolated $\mathrm{CABG}$ & - & $5 y$ & - \\
\hline van Dijk (Octopus), ${ }^{2} 2007$ & $1998-2000$ & Primary isolated $\mathrm{CABG}$ & - & $5 y$ & - \\
\hline Puskas (SMART), ${ }^{24} 2011$ & March 2000 to August 2001 & $\begin{array}{l}\text { Elective primary isolated CABG } \\
\text { for multivessel disease }\end{array}$ & - & Mean, 7.5 y (range, 6.8-8.4) & - \\
\hline \multicolumn{6}{|l|}{ Adjusted observational study } \\
\hline Brown, ${ }^{\text {E1 }} 2008$ & January 2000 to June 2004 & Nonemergency isolated CABG & Multivariable CPHR & $6 \mathrm{y}$ (median, 4.1) & - \\
\hline Bakaeen (CICSP), ${ }^{\mathrm{E} 2} 2013$ & October 1997 to April 2011 & Primary isolated $\mathrm{CABG}$ & PS matching & Median, 6.68 y (IQR, 3.72-9.35) & - \\
\hline Cooper, ${ }^{\mathrm{E} 3} 2009$ & January 1997 to March 2007 & Primary isolated CABG & PS-adjusted CPHR & $10 \mathrm{y}$ & $\begin{array}{l}\text { White } \\
\text { Black } \\
\text { Total }\end{array}$ \\
\hline Di Mauro, ${ }^{\mathrm{E} 4} 2007$ & November 1994 to December 2001 & $\begin{array}{l}\text { Isolated CABG for multivessel } \\
\text { disease }\end{array}$ & PS matching & $7.5 \pm 1.9 y$ & - \\
\hline Filardo, ${ }^{\text {E5 }} 2011$ & $1997-2008$ & Isolated $\mathrm{CABG}$ & PS-adjusted CPHR & $10 \mathrm{y}$ & - \\
\hline $\mathrm{Fu},{ }^{\mathrm{E} 6} 2009$ & $1999-2005$ & Isolated CABG & PS-adjusted CPHR & $57.96 \pm 23.46 \mathrm{mo}$ & $\begin{array}{c}\text { Men } \\
\text { Women } \\
\text { Total }\end{array}$ \\
\hline García Fuster, ${ }^{\text {E7 }} 2013$ & January 1995 to June 2011 & Elective primary isolated $\mathrm{CABG}$ & PS matching & $\begin{array}{l}77 \pm 53 \text { mo, off-pump; } \\
126 \pm 54 \text { mo, on-pump }\end{array}$ & - \\
\hline Gorki, $^{\mathrm{E} 8} 2010$ & January 1999 to September 2008 & $\begin{array}{l}\mathrm{EF} \leq 30 \% \text {; isolated coronary } \\
\quad \text { disease }\end{array}$ & PS matching & $\begin{array}{l}10 \text { y (median, } 44 \text { mo; range, } \\
\text { 0-120, off-pump; median, } \\
64.8 \text { mo; range, } 0-120, \\
\text { on-pump) }\end{array}$ & - \\
\hline $\mathrm{Hu},{ }^{\mathrm{E} 9} 2010$ & 1999-2006 & Isolated CABG & PS matching & $8 \mathrm{y}($ mean, 4.5$)$ & - \\
\hline Locker, ${ }^{\mathrm{E} 10} 2013$ & 1993-2009 & $\begin{array}{l}\text { Primary isolated CABG for } \\
\text { multivessel disease }\end{array}$ & Multivariable CPHR & $7.6 \pm 4.6 \mathrm{y}$ & - \\
\hline Murzi, ${ }^{\mathrm{E} 11} 2012$ & April 1996 to December 2009 & $\begin{array}{l}\text { Isolated CABG for left main } \\
\text { disease }\end{array}$ & PS matching & $\begin{array}{l}10 \mathrm{y}(50.5 \pm 31.2 \mathrm{mo}, \\
\text { off-pump; } 54.4 \pm 34.1 \mathrm{mo}, \\
\text { on-pump) }\end{array}$ & - \\
\hline Wu (NY CSRS), ${ }^{\mathrm{E} 12} 2012$ & July-December 2000 & Isolated CABG & PS matching & Median, 7.2 y (IQR, 7.0-7.4) & - \\
\hline $\mathrm{Raja}^{\mathrm{E} 13} 2013$ & January-December 2002 & $\begin{array}{l}\text { Isolated CABG for multivessel } \\
\text { disease }\end{array}$ & PS matching & $10 \mathrm{y}$ & - \\
\hline Robertson, ${ }^{\mathrm{E} 14} 2013$ & January 1997 to June 2003 & Primary isolated $\mathrm{CABG}$ & PS matching & Median, $5.9 \mathrm{y}$ & - \\
\hline Sarin, ${ }^{\text {E15 }} 2011$ & January 1996 to September 2008 & Isolated CABG & PS-adjusted CPHR & $10 \mathrm{y}$ & - \\
\hline $\begin{array}{l}\text { Dalén (SWEDEHEART), }{ }^{\text {E16 }} \\
2013\end{array}$ & $1998-2008$ & CABG in Sweden & PS matching & Mean, 7.1 y; 362,254 pt-y & - \\
\hline Synnergren, ${ }^{\mathrm{E} 17} 2008$ & $1995-2004$ & Isolated CABG & $\begin{array}{l}\text { Multivariable } \\
\text { Poisson regression }\end{array}$ & $5.0 \pm 2.8$ y (range, $0.5-10.5)$ & - \\
\hline
\end{tabular}

$\overline{D M}$, Diabetes mellitus; BHACAS, Beating Heart Against Cardioplegic Arrest Studies; $M I$, myocardial infarction; $E F$, ejection fraction; $C A B G$, coronary artery bypass grafting; $I Q R$, interquartile range; MASS, Medicine, Angioplasty, or Surgery Study; SMART, Surgical Management of Arterial Revascularization Therapies; CPHR, Cox proportional hazards regression; CICSP, Continuous Improvement in Cardiac Surgery Program; PS, propensity score; NY CSRS, New York State's Cardiac Surgery Reporting System; SWEDEHEART, Swedish Web-system for Enhancement and Development of Evidence-based care in Heart Disease Evaluated According to Recommended Therapies; $p t-y$, patient-years. *Data from Angelini et al. ${ }^{3}$ 
was still statistically significant. In general, exclusion of any single study from the analysis did not substantively alter the overall result of our analysis (Figure 2). Additionally, pooling 10 PS-matched studies ${ }^{\text {E2,E4,E7-E9,E11-E14,E16 }}$ (56,028 patients) did not substantially change the pooled estimate (fixed-effects HR, 1.05 ; $95 \%$ CI, 1.004 to 1.09 ; $P$ for overall effect $=.03 ; P$ for heterogeneity $=.51$ ).

\section{Publication Bias}

To assess the publication bias, we generated a funnel plot of the logarithm of the effect size (HR) versus the precision (reciprocal of the standard error) for each study (Figure 3). No evidence was found of a significant publication bias (2-tailed $P=.87$ and $P=.55$, adjusted rank correlation and linear regression test, respectively).

TABLE 1. Continued

\begin{tabular}{|c|c|c|c|c|c|c|c|}
\hline \multicolumn{4}{|c|}{ Off-pump } & \multicolumn{4}{|c|}{ On-pump } \\
\hline Patients (n) & Age (y) & Women $(\%)$ & DM $(\%)$ & Patient (n) & Age (y) & Women (\%) & DM $(\%)$ \\
\hline 100 & $62.2 \pm 9.6$ & 18.0 & 19.0 & 100 & $61.7 \pm 8.6$ & 21.0 & 14.0 \\
\hline 100 & $63.8 \pm 8.5$ & 18.0 & 32.0 & 101 & $61.2 \pm 9.2$ & 14.9 & 29.7 \\
\hline 200 & $63.0 \pm 9.1$ & 18.0 & 25.5 & 201 & $61.4 \pm 8.9$ & 17.9 & 21.9 \\
\hline 149 & $62.2 \pm 10.0$ & 18.8 & 29.5 & 150 & $63.7 \pm 10.0$ & 20.0 & 36.0 \\
\hline 155 & 61 & 22 & 29 & 153 & 59 & 20 & 27 \\
\hline 142 & $61.7 \pm 9.2$ & 33.8 & 9.2 & 139 & $60.8 \pm 8.8$ & 29.5 & 16.5 \\
\hline 98 & $62.5 \pm 9.5$ & 22.4 & 32.7 & 99 & $62.2 \pm 11.1$ & 23.2 & 33.3 \\
\hline 733 & - & 28.1 & - & 778 & - & 25.1 & - \\
\hline 8911 & $63.9 \pm 9.3$ & 0.9 & 39.3 & 26,733 & $63.9 \pm 8.9$ & 1.0 & 40.1 \\
\hline 4970 & $63.7 \pm 11.2$ & 28.8 & 33.2 & 5871 & $62.9 \pm 10.6$ & 25.4 & 33.9 \\
\hline 1086 & $60.4 \pm 11.7$ & 45.7 & 47.0 & 947 & $59.6 \pm 11.1$ & 38.2 & 46.7 \\
\hline 6056 & $63.1 \pm 11.4$ & 31.9 & 35.7 & 6818 & $62.4 \pm 10.7$ & 27.2 & 35.7 \\
\hline 862 & $63.9 \pm 9.5$ & 16.5 & 23.5 & 862 & $64.2 \pm 9.1$ & 17.9 & 23.5 \\
\hline 732 & $64.9 \pm 10.8$ & 31.4 & 33.2 & 7349 & $64.4 \pm 10.7$ & 27.6 & 34.7 \\
\hline 2007 & $60.35 \pm 9.36$ & 0 & 24.7 & 2460 & $59.61 \pm 7.69$ & 0 & 23.4 \\
\hline 403 & $62.90 \pm 8.18$ & 100 & 31.5 & 489 & $60.74 \pm 6.94$ & 100 & 32.9 \\
\hline 2410 & $60.78 \pm 9.22$ & 16.7 & 25.9 & 2949 & $59.80 \pm 7.58$ & 16.6 & 25.0 \\
\hline 250 & $66 \pm 9$ & 22.0 & 41.6 & 250 & $64 \pm 9$ & 18.8 & 33.6 \\
\hline 346 & $66.5 \pm 10.6$ & 20.8 & 50.0 & 346 & $64.7 \pm 11.0$ & 23.7 & 42.8 \\
\hline 2088 & - & - & - & 2088 & - & - & - \\
\hline 366 & - & - & - & 8256 & - & - & - \\
\hline 548 & $65.7 \pm 9.3$ & 19.9 & 20.8 & 548 & $66.2 \pm 8.7$ & 19.2 & 20.6 \\
\hline 2631 & $67.7 \pm 11.0$ & 29.8 & 31.6 & 2631 & $67.7 \pm 10.7$ & 29.3 & 30.9 \\
\hline 307 & $62.3 \pm 11.8$ & 28.7 & 35.2 & 307 & $62.6 \pm 7.9$ & 31.3 & 32.2 \\
\hline 308 & $63.5 \pm 10.6$ & 25.3 & 27.6 & 308 & $62.7 \pm 10.6$ & 27.3 & 26.6 \\
\hline 540 & $82.9 \pm 2.8$ & 45.9 & 28.0 & 397 & $82.3 \pm 2.4$ & 44.6 & 27.2 \\
\hline 2852 & Mean, 65.4 & 26 & 18 & 2852 & Mean, 66.1 & 25 & 20 \\
\hline 947 & $64 \pm 10$ & 27.0 & 17.8 & 8461 & $66 \pm 9$ & 21.1 & 20.4 \\
\hline
\end{tabular}


TABLE 2. Details of revascularization

\begin{tabular}{|c|c|c|c|c|c|c|c|}
\hline \multirow[b]{2}{*}{ Study } & \multirow[b]{2}{*}{ Subgroup } & \multicolumn{3}{|c|}{ Graft/patient (n) } & \multicolumn{3}{|c|}{ Index of completeness of revascularization } \\
\hline & & Off-pump & On-pump & $P$ value & Off-pump & On-pump & $P$ value \\
\hline \multicolumn{8}{|l|}{ Randomized controlled trial } \\
\hline \multirow[t]{2}{*}{$\begin{array}{l}\text { Angelini (BHACAS } 1 \\
\text { and } 2),{ }^{20} 2009\end{array}$} & BHACAS $1 *$ & Median, 2 (range, 1-4) & $\begin{array}{l}\text { Median, } 2 \\
\quad(\text { range, 1-4) }\end{array}$ & .3 & - & - & - \\
\hline & $\begin{array}{c}\text { BHACAS } 1 \\
\text { and } 2 \dagger\end{array}$ & $\begin{array}{l}\geq 3 \text { grafts in patients } \\
\text { of } 45.0 \%\end{array}$ & $\begin{array}{l}\geq 3 \text { grafts in } \\
\text { patients of } 56.2 \%\end{array}$ & NS & - & - & - \\
\hline Karolak, ${ }^{21} 2007$ & - & $\begin{array}{l}\text { Anastomosis; median, } \\
2.8 \pm 0.9\end{array}$ & $\begin{array}{l}\text { Anastomosis; } \\
\quad \text { median, } 3.0 \pm 0.9\end{array}$ & .06 & - & - & - \\
\hline $\begin{array}{l}\text { Hueb (MASS III), }{ }^{15} \\
2010\end{array}$ & - & $\begin{array}{l}2.49 \text { (anastomosis, } \\
2.60 \text { ) }\end{array}$ & $\begin{array}{l}2.97 \text { (anastomosis, } \\
\text { 3.18) }\end{array}$ & $<.001$ & - & - & - \\
\hline $\begin{array}{l}\text { van Dijk (Octopus), }{ }^{2} \\
2007\end{array}$ & - & $\begin{array}{l}\text { Anastomosis; median, } \\
2.4 \pm 1.0 \ddagger\end{array}$ & $\begin{array}{l}\text { Anastomosis; } \\
\quad \text { median, } 2.6 \pm 1.0 \ddagger\end{array}$ & .05 & - & - & - \\
\hline $\begin{array}{l}\text { Puskas (SMART), }{ }^{24} \\
\quad 2011\end{array}$ & - & 3.39 & 3.40 & - & 1.01 & 1.00 & - \\
\hline \multicolumn{8}{|l|}{ Adjusted observational study } \\
\hline Brown, ${ }^{\mathrm{E} 1} 2008$ & - & 3.0 & 3.3 & $<.001$ & - & - & - \\
\hline $\begin{array}{l}\text { Bakaeen (CICSP), } \\
\quad 2013\end{array}$ & - & $\begin{array}{l}2.66 \pm 1.03(\geq 3 \\
\text { anastomoses in } \\
\text { patients of } 56.3 \%)\end{array}$ & $\begin{array}{l}3.18 \pm 0.89(\geq 3 \\
\text { anastomoses in } \\
\text { patients, } 79.5 \%)\end{array}$ & $<.0001$ & - & - & - \\
\hline Cooper, ${ }^{\mathrm{E} 3} 2009$ & - & - & - & - & - & - & - \\
\hline Di Mauro, ${ }^{\mathrm{E} 4} 2007$ & - & $\begin{array}{l}\text { Anastomosis; median, } \\
2.6 \pm 0.7\end{array}$ & $\begin{array}{l}\text { Anastomosis; } \\
\quad \text { median, } 2.6 \pm 0.7\end{array}$ & .717 & - & - & - \\
\hline Filardo, ${ }^{\text {E5 }} 2011$ & - & - & - & - & - & - & - \\
\hline \multirow[t]{2}{*}{$\mathrm{Fu},{ }^{\mathrm{E} 6} 2009$} & Men & $\begin{array}{l}\text { Saphenous and arterial } \\
\text { anastomoses, } \\
1.90 \pm 0.96 \text { and } \\
1.09 \pm 0.52 \\
\text { respectively }\end{array}$ & $\begin{array}{l}\text { Saphenous and } \\
\text { arterial } \\
\text { anastomoses, } \\
2.75 \pm 1.01 \text { and } \\
1.07 \pm 0.50, \\
\text { respectively }\end{array}$ & - & $1.1108 \pm 0.3188$ & $1.3556 \pm 0.3265$ & $<.0001$ \\
\hline & Women & $\begin{array}{l}\text { Saphenous and arterial } \\
\text { anastomoses, } \\
1.90 \pm 0.95 \text { and } \\
0.95 \pm 0.41 \\
\text { respectively }\end{array}$ & $\begin{array}{l}\text { Saphenous and } \\
\text { arterial } \\
\text { anastomoses, } \\
2.70 \pm 0.95 \text { and } \\
0.94 \pm 0.44, \\
\text { respectively }\end{array}$ & - & $1.0544 \pm 0.2813$ & $1.2962 \pm 0.3564$ & $<.0001$ \\
\hline García Fuster, ${ }^{\mathrm{E} 7} 2013$ & - & $2.7 \pm 1.0$ & $2.8 \pm 0.9$ & .35 & - & - & - \\
\hline Gorki, ${ }^{\mathrm{E} 8} 2010$ & - & $3.10 \pm 0.88$ & $3.53 \pm 1.02$ & - & - & - & - \\
\hline $\mathrm{Hu},{ }^{\mathrm{E} 9} 2010$ & - & - & - & - & $\begin{array}{l}1.1 \pm 0.3 \text { (complete } \\
\quad \text { in patients, } 87.2 \%) \S\end{array}$ & $\begin{array}{l}1.3 \pm 0.3 \\
\quad(\text { complete } \\
\text { in patients, } \\
96.0 \%) \S\end{array}$ & $<.001$ \\
\hline Locker, ${ }^{\mathrm{E} 10} 2013$ & - & - & - & - & - & - & - \\
\hline Murzi, $^{\text {E11 }} 2012$ & - & $2.7 \pm 0.7$ & $3 \pm 0.7$ & .001 & $\begin{array}{l}1 \pm 0.3 \text { (complete } \\
\quad \text { in patients, } 88.3 \% \text { ) }\end{array}$ & $\begin{array}{l}1.1 \pm 0.3 \\
\text { (complete } \\
\text { in patients, } \\
92.0 \%)\end{array}$ & $.001(.04)$ \\
\hline $\begin{array}{l}\text { Wu (NY CSRS), }{ }^{\mathrm{E} 12} \\
2012\end{array}$ & - & $\begin{array}{l}\text { Anastomosis; median, } \\
\quad 2.69 \S\end{array}$ & $\begin{array}{l}\text { Anastomosis; } \\
\text { median, } 3.34 \S\end{array}$ & $<.001$ & - & - & - \\
\hline Raja $^{\mathrm{E} 13} 2013$ & - & $2.91 \pm 1.06 \S$ & $3.4 \pm 0.4 \S$ & $<.01$ & $1.09 \pm 0.17 \S$ & $1.11 \pm 0.19 \S$ & .87 \\
\hline Robertson, ${ }^{\text {E14 }} 2013$ & - & $\begin{array}{c}\geq 3 \text { Anastomoses in } \\
\text { patients, } 51.9 \%\end{array}$ & $\begin{array}{c}\geq 3 \text { Anastomoses in } \\
\text { patients, } 68.5 \%\end{array}$ & .0001 & $\begin{array}{c}\text { Complete in patients, } \\
79.2 \%\end{array}$ & $\begin{array}{l}\text { Complete in } \\
\text { patients, } 88.3 \%\end{array}$ & .002 \\
\hline Sarin, ${ }^{\mathrm{E} 15} 2011$ & - & - & - & - & - & - & - \\
\hline $\begin{array}{l}\text { Dalén } \\
\text { (SWEDEHEART), } \\
2013\end{array}$ & - & $2.0 \pm 1.0 \S$ & $3.5 \pm 1.0 \S$ & $<.001$ & - & - & - \\
\hline
\end{tabular}


TABLE 2. Continued

\begin{tabular}{|c|c|c|c|c|c|c|c|}
\hline \multirow[b]{2}{*}{ Study } & \multirow[b]{2}{*}{ Subgroup } & \multicolumn{3}{|c|}{ Graft/patient (n) } & \multicolumn{3}{|c|}{ Index of completeness of revascularization } \\
\hline & & Off-pump & On-pump & $P$ value & Off-pump & On-pump & $P$ value \\
\hline Synnergren, ${ }^{\mathrm{E} 17} 2008$ & - & - & - & - & $\begin{array}{l}\text { Complete in } \\
\text { patients, } 67.1 \%\end{array}$ & $\begin{array}{l}\text { Complete in } \\
\text { patients, } 82.9 \%\end{array}$ & $<.001$ \\
\hline
\end{tabular}

BHACAS, Beating Heart Against Cardioplegic Arrest Studies; NS, not significant; MASS, Medicine, Angioplasty, or Surgery Study; SMART, Surgical Management of Arterial Revascularization Therapies; CICSP, Continuous Improvement in Cardiac Surgery Program; NY CSRS, New York State's Cardiac Surgery Reporting System; SWEDEHEART, Swedish Web-system for Enhancement and Development of Evidence-based care in Heart Disease Evaluated According to Recommended Therapies. *Data from Ascione R, Caputo M, Calori G, Lloyd CT, Underwood MJ, Angelini GD. Predictors of atrial fibrillation after conventional and beating heart coronary surgery: A prospective, randomized study. Circulation. 2000;102:1530-5. †Data from Angelini et al. ${ }^{3} \ddagger$ Data from van Dijk D, Nierich AP, Jansen EW, Nathoe HM, Suyker WJ, Diephuis JC, et al, Octopus Study Group. Early outcome after off-pump versus on-pump coronary bypass surgery: results from a randomized study. Circulation. 2001;104:1761-6. §Unmatched patients.

\section{DISCUSSION}

The results of our analysis suggest that off-pump CABG might be associated with worse long-term ( $\geq 5$-year) survival compared with on-pump CABG. The results from the adjusted observational studies were the most compelling, with data from 102,820 patients in 17 different studies demonstrating a 7\% increase in all-cause mortality with off-pump relative to one-pump CABG that was robust in the sensitivity analyses, even after eliminating the highest weight and largest size, ${ }^{\mathrm{E} 2}$ second highest weight, ${ }^{\mathrm{E} 12}$ or second largest size study. ${ }^{\mathrm{E} 3}$ The data from 5 randomized controlled trials were less robust, however, likely owing to the systematic underpowering of these trials in the design phase and, primarily, the small number of enrolled patients $(\mathrm{n}=1486)$. The observed $14 \%$ increase (underpowered as reflected by the wide 95\% CIs and

TABLE 3. Graft patency and cardiac events

\begin{tabular}{|c|c|c|c|c|}
\hline \multirow[b]{2}{*}{ Outcome } & \multirow[b]{2}{*}{ Study } & \multicolumn{3}{|c|}{ Event rate $(\%)$} \\
\hline & & Off-pump & On-pump & $P$ value \\
\hline \multicolumn{5}{|l|}{ Graft patency } \\
\hline & BHACAS 1 and $2,,^{20} 2009$ & & & \\
\hline & SMART, $^{24} 2011$ & $89.0^{*}, \dagger$ & $89.4^{*}, \dagger$ & $>.99$ \\
\hline & & $76^{*}, \dagger$ & $83.5^{*}, \dagger$ & .44 \\
\hline \multicolumn{5}{|l|}{ Cardiac mortality } \\
\hline & Octopus, ${ }^{2} 2007$ & $0 \dagger$ & $1.4 \dagger$ & .24 \\
\hline & $\mathrm{Hu},{ }^{\mathrm{E9}} 2010$ & $5.9 \ddagger, \S$ & $6.1 \neq, \S$ & .54 \\
\hline \multicolumn{5}{|l|}{ Repeat revascularization } \\
\hline & BHACAS 1 and $2,,^{20} 2009$ & $1.5 \dagger$ & $1.5 \dagger$ & $1.00 \|$ \\
\hline & MASS III, ${ }^{15} 2010$ & $6.5 \dagger$ & $5.9 \dagger$ & .84 \\
\hline & Octopus, ${ }^{2} 2007$ & $7.7 \dagger$ & $5.0 \dagger$ & .47 \\
\hline & SMART, $^{24} 2011$ & $2.3 \dagger$ & $2.3 \dagger$ & 1.0 \\
\hline & $\mathrm{Hu},{ }^{\mathrm{E} 9} 2010$ & $9.1 \neq, \S$ & $8.4+, \S$ & .03 \\
\hline & Raja, ${ }^{\text {E13 }} 2013$ & $0.7 \S$ & $0.7 \S$ & 1.00 \\
\hline \multicolumn{5}{|l|}{ Angina } \\
\hline & BHACAS 1 and $2,,^{20} 2009$ & $14.0^{\dagger}$ & $18.9 \dagger$ & $.19 \|$ \\
\hline & MASS III, ${ }^{15} 2010$ & $11.8 \dagger$ & $6.7 \dagger$ & .09 \\
\hline & SMART,$^{24} 2011$ & $25.6 \dagger$ & $11.4 \dagger$ & .09 \\
\hline & $\mathrm{Hu},{ }^{\mathrm{E} 9} 2010$ & $28.7 \neq, \S$ & $24.7 \ddagger, \S$ & .002 \\
\hline \multicolumn{5}{|l|}{ Myocardial infarction } \\
\hline & BHACAS 1 and $2,{ }^{20} 2009$ & $4.0^{\dagger}$ & $4.0^{\dagger}$ & $.99 \|$ \\
\hline & MASS III, ${ }^{15} 2010$ & $6.5+, \uparrow$ & $2.0+, \uparrow$ & .05 \\
\hline & Octopus, ${ }^{2} 2007$ & $4.9 \dagger$ & $6.5^{\dagger}$ & .62 \\
\hline & $\mathrm{Hu},{ }^{\mathrm{E9}} 2010$ & $3.3+, \S, \#$ & $2.8+, \S, \#$ & .22 \\
\hline \multicolumn{5}{|c|}{ Rehospitalization for cardiac reason } \\
\hline & Karolak, ${ }^{21} 2007$ & $35 \dagger$ & $35 \dagger$ & .57 \\
\hline & $\mathrm{Hu},{ }^{\mathrm{E} 9} 2010$ & $45.2 \ddagger, \S$ & $37.5 \ddagger, \S$ & $<.001$ \\
\hline & Raja, ${ }^{\mathrm{E} 13} 2013$ & $3.3 \S$ & $3.6 \S$ & .93 \\
\hline & Robertson, ${ }^{\text {E14 }} 2013$ & $31.7 \S$ & $31.9 \S$ & .72 \\
\hline & SWEDEHEART, ${ }^{\text {E16 }} 2013$ & $51 \S$, ** & $57 \S, * *$ & .22 \\
\hline
\end{tabular}

BHACAS, Beating Heart Against Cardioplegic Arrest Studies; SMART, Surgical Management of Arterial Revascularization Therapies; MASS, Medicine, Angioplasty, or Surgery Study; SWEDEHEART, Swedish Web-system for Enhancement and Development of Evidence-based care in Heart Disease Evaluated According to Recommended Therapies. *Patency rate. †Randomized patients. $\ddagger$ Per 1000 person-years. §Propensity-score matched patients. ||Calculated by us. $\uparrow$ Acute myocardial infarction. \#Nonfatal myocardial infarction. **Composite of all-cause mortality and rehospitalization for myocardial infarction, heart failure, or stroke. 


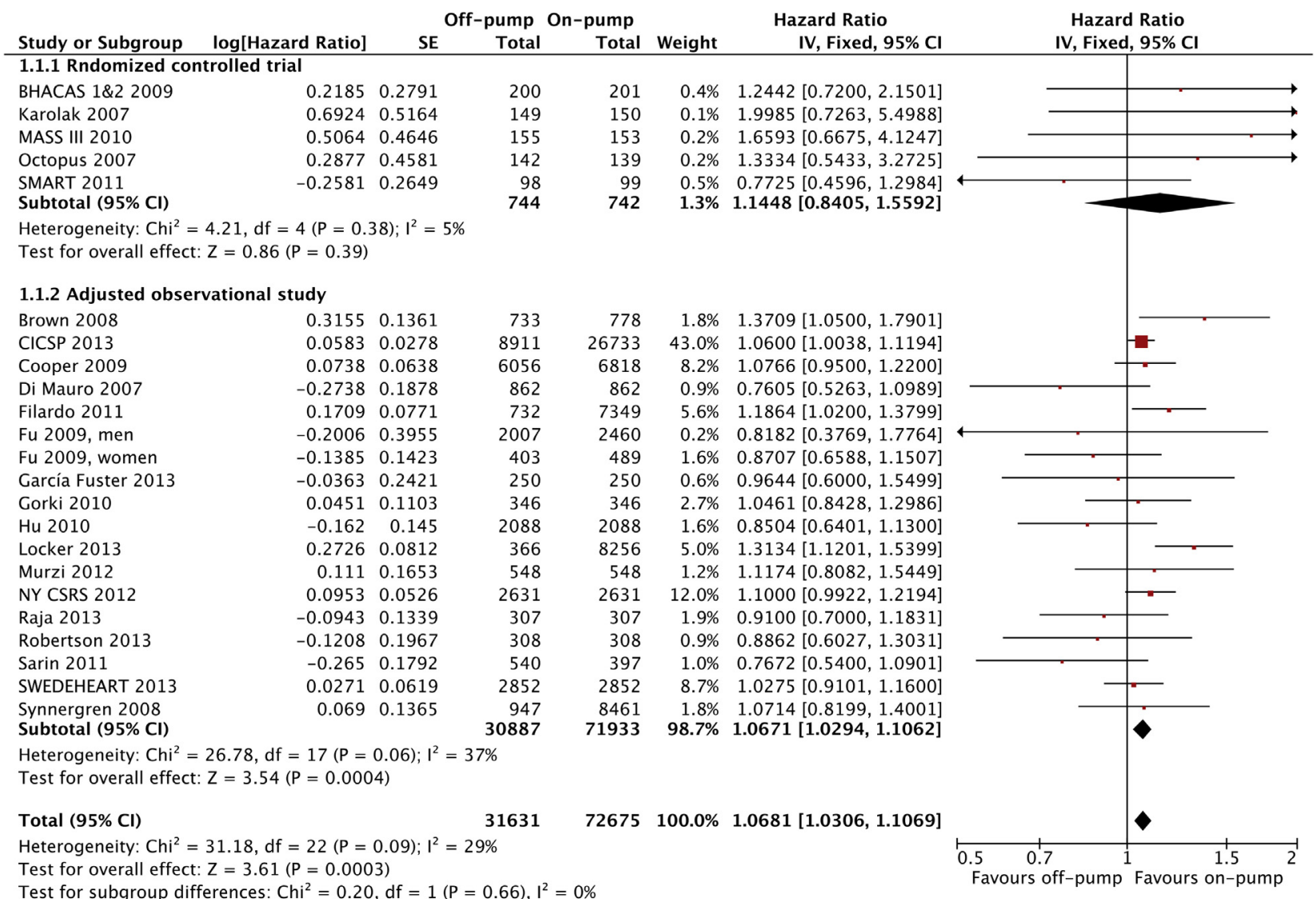

FIGURE 1. Forest plot of hazard ratios for long-term all-cause mortality among patients assigned to off-pump versus on-pump coronary artery bypass grafting. SE, Standard error; IV, inverse variance; $C I$, confidence interval; BHACAS, Beating Heart Against Cardioplegic Arrest Studies; MASS, Medicine, Angioplasty, or Surgery Study; SMART, Surgical Management of Arterial Revascularization Therapies; CICSP, Continuous Improvement in Cardiac Surgery Program; NY CSRS, New York State's Cardiac Surgery Reporting System; SWEDEHEART, Swedish Web-system for Enhancement and Development of Evidence-based care in Heart Disease Evaluated According to Recommended Therapies.

lack of statistical significance) in $\geq 5$-year mortality in the patients undergoing off-pump CABG is far lower than the $34 \%$ increase in $>30$-day mortality (risk ratio [RR], 1.34; 95\% CI, 1.08-1.67; $P=.009)$ and $35 \%$ increase in $\geq 1$-year mortality (odds ratio, $1.35 ; 95 \% \mathrm{CI}$, $1.07-1.70 ; P=.01)$ demonstrated in the recent Cochrane systematic review ${ }^{1}$ and our previous meta-analysis, ${ }^{19}$ respectively.

In off-pump versus on-pump CABG, the best evidence of a lower number of distal anastomoses ${ }^{1}$ and the rate of graft patency $^{25}$ could explain the worse long-term survival demonstrated in the present meta-analysis. This is because CABG with complete revascularization improves survival compared with $\mathrm{CABG}$ with incomplete revascularization in patients with multivessel disease. ${ }^{26}$ The average mean difference in the number of distal anastomoses after off-pump CABG was -0.28 (95\% CI, -0.40 to -0.16 ; $P<.00001)$ in the Cochrane meta-analysis ${ }^{1}$ of 57 trials (7071 participants). A recent 2014 meta-analysis by Zhang and colleagues ${ }^{25}$ of 12 randomized controlled trials (3894 and 4137 grafts performed during off-pump CABG and on-pump CABG, respectively) showed an increased risk of occlusion of all grafts (RR, 1.35; 95\% CI, 1.16-1.57; $P<.001)$ and saphenous vein grafts (RR, $1.41 ; 95 \% \mathrm{CI}$, $1.24-1.60 ; P<.001)$ in the off-pump group. However, no significant difference was found in graft occlusion of the left internal mammary artery (RR, $1.15 ; 95 \% \mathrm{CI}$, $0.83-1.59 ; P=.407)$ or radial artery $(\mathrm{RR}, 1.37 ; 95 \% \mathrm{CI}$, $0.76-2.47 ; P=.298)$ grafts between off pump and onpump CABG. However, our recent 2014 meta-analysis ${ }^{26}$ of adjusted HRs (not unadjusted) from 14 observational studies $(30,389$ patients) demonstrated a statistically significant $37 \%$ reduction in follow-up mortality with complete revascularization relative to incomplete revascularization (HR, 0.63; 95\% CI, 0.53-0.75; $P<.00001$ ). Furthermore, the finding of worse long-term survival after off-pump relative to on-pump CABG might be strengthened by the results from another 2013 meta-analysis. 27 The pooled analysis of 12 randomized trials enrolling a total of 11,594 patients demonstrated a statistically significant 


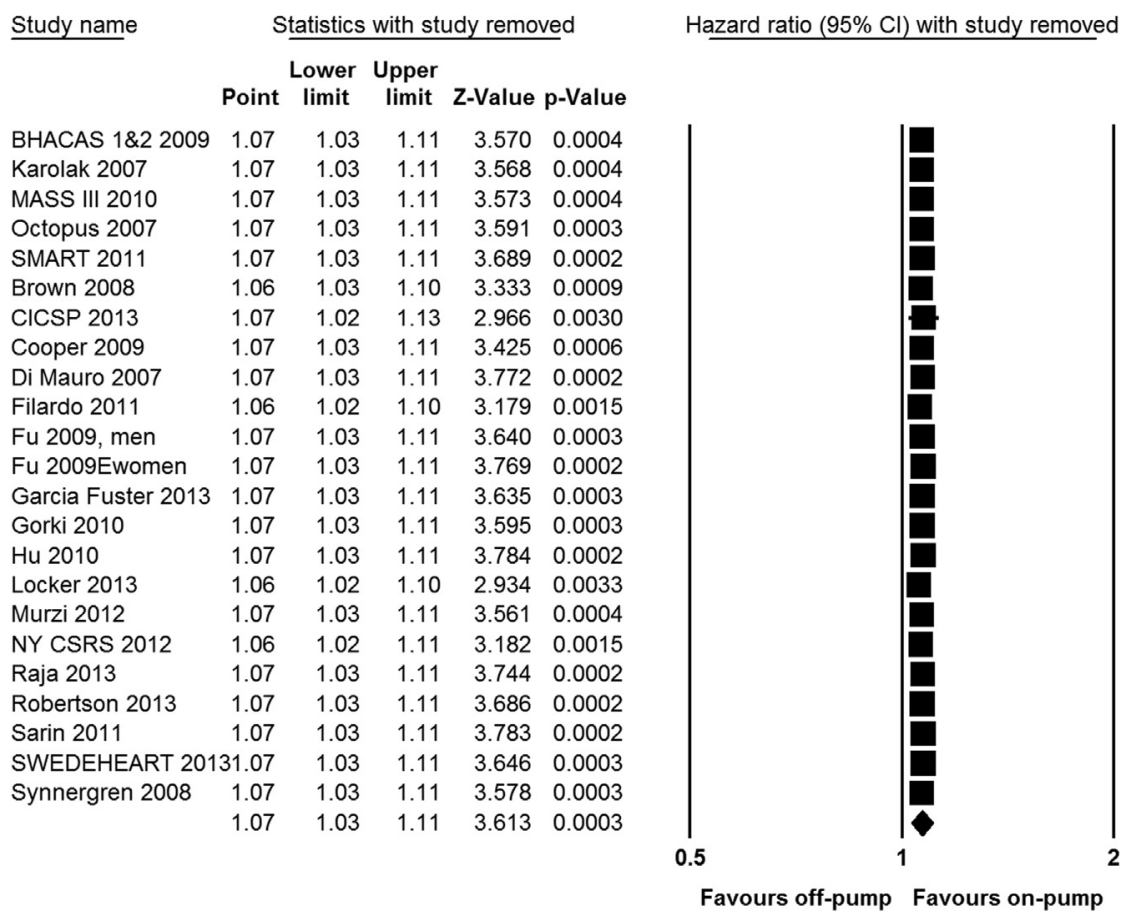

FIGURE 2. One-study-removed meta-analysis of hazard ratios for all-cause long-term mortality among patients assigned to off-pump versus on-pump coronary artery bypass grafting. CI, Confidence interval; BHACAS, Beating Heart Against Cardioplegic Arrest Studies; MASS, Medicine, Angioplasty, or Surgery Study; SMART, Surgical Management of Arterial Revascularization Therapies; CICSP, Continuous Improvement in Cardiac Surgery Program; NY CSRS, New York State's Cardiac Surgery Reporting System; SWEDEHEART, Swedish Web-system for Enhancement and Development of Evidence-based care in Heart Disease Evaluated According to Recommended Therapies.

$38 \%$ increase in repeat revascularization rates with off-pump relative to on-pump CABG (odds ratio, 1.38; $95 \% \mathrm{CI}, 1.09-1.76 ; P=.008)^{27}$

Successful performance of off-pump CABG seems likely to depend on initial technical risks more than with on-pump CABG, because, inherently, on a beating heart, performing delicate anastomoses is difficult and the potential degree of revascularization completeness or quality low. ${ }^{28}$ Thus, to guarantee surgeon' skill in the assigned technique (off-pump or on-pump CABG), the approach of an expertise-based randomized controlled trial ${ }^{29}$ should be used. In the largest size (4752 patients) randomized controlled trial, the CABG Off or On Pump Revascularization Study, ${ }^{28}$ expertise was defined as $>2$ years of experience and the completion of $>100$ procedures involving the specific technique. Those surgeons who had met these criteria for each type of operation separately were considered to have expertise in both techniques and were allowed to perform both types of CABG during the trial. ${ }^{28}$ The investigators of the trial ${ }^{30}$ found, at 1 year, no significant differences between the 2 groups in the rate of death (HR, 0.87; 95\% CI, 0.58-1.31), nonfatal stroke, nonfatal myocardial infarction, or nonfatal new renal failure requiring dialysis or in the rate of subsequent revascularization procedures. Also, in the second largest size (2370 patients) randomized trial, German Off-Pump Coronary Artery Bypass Grafting in Elderly Patients, ${ }^{31}$ the study surgeons were required to be established experts in the performance of either off-pump or on-pump CABG and the average number of CABG surgeries performed before the study was 514 off-pump (median, 322) for the off-pump CABG surgeons and 1378 on-pump (median, 578) for the on-pump CABG surgeons. The trial showed no significant differences between off-pump and on-pump CABG with regard to death (HR, $0.88 ; 95 \%$ CI, $0.65-1.18 ; P=.38$ ), stroke, myocardial infarction, repeat revascularization, or new renal replacement therapy within 12 months after surgery. ${ }^{31}$ The mid-term and long-term outcomes of these large-size expertise-based randomized trials are expected.

Our analysis must be viewed in the context of its limitations. First, we used data from adjusted observational studies and randomized controlled trials. Although the study design of the randomized trials, which balance both known and unknown confounders across treatment groups, is the least vulnerable to bias, the patients enrolled in them might not be representative of the patients typically seen in clinical practice. However, because the potential biases must be greater for observational studies than for the randomized trials, the results should be always interpreted 


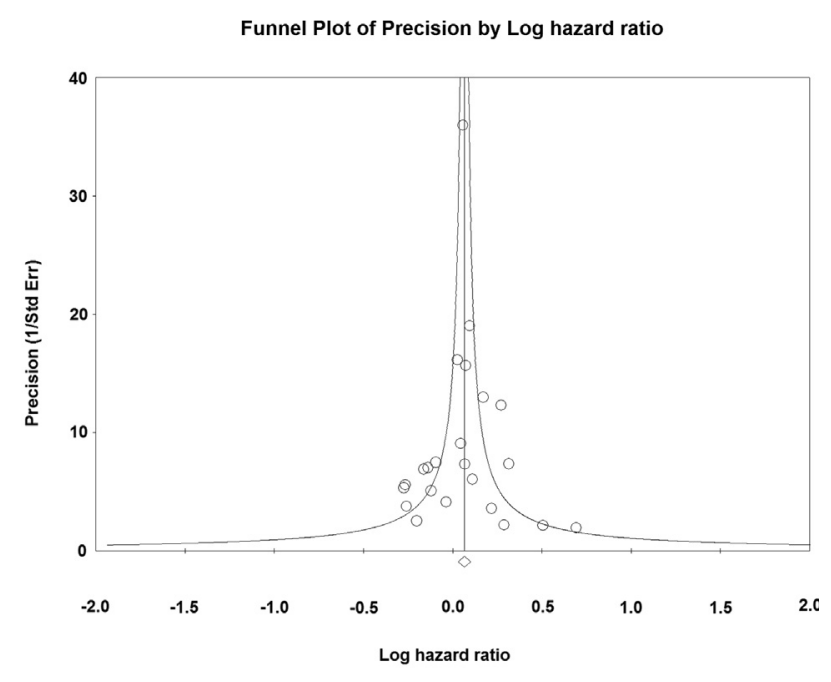

FIGURE 3. Funnel plot of the logarithm of effect size (hazard ratio) versus the precision (reciprocal of the standard error [Std Err]) for each study.

with caution when they have been included in metaanalyses. ${ }^{32}$ Particular concerns arise in terms of differences between patients in different intervention groups (selection bias). In contrast to randomized trials, it would usually be appropriate to analyze adjusted (ie, attempting to control for confounding), rather than unadjusted, effect estimates. ${ }^{32}$ To reduce the effect of treatment-selection bias and potential confounding in observational studies, rigorous adjustment for significant differences in the baseline characteristics of the patients should be conducted. Furthermore, adjusted estimates (not unadjusted) should be pooled in a meta-analysis that includes observational studies. In the present meta-analysis, we strictly abstracted (and then combined in a meta-analysis) exclusive adjusted relative risk estimates (not unadjusted) from the observational studies. Second, our results could have been influenced by a publication bias favoring on-pump CABG. This risk was minimized through an exhaustive search of the available published data. Although the statistical test results did not indicate a publication bias, we clearly had limited power to detect such a bias, given the small number of studies examined.

\section{CONCLUSIONS}

We found that, from a meta-analysis of 22 studies enrolling a total of $>100,000$ patients, off-pump CABG is likely to be associated with worse long-term ( $\geq 5$-year) survival compared with on-pump CABG. Because a long-term mortality reduction must imply the greatest clinical benefit among patients undergoing $\mathrm{CABG}$, on-pump rather than off-pump CABG should be considered for patients without contraindications to cardiopulmonary bypass. The longterm results of large $(>2000$ patients $)$ randomized controlled trials, such as the CABG Off or On Pump Revascularization Study, ${ }^{30}$ German Off-Pump Coronary Artery Bypass Grafting in Elderly Patients, ${ }^{31}$ and Randomized On/Off Bypass ${ }^{14}$ study, will render the last judgment for the question of whether to pump or not to pump.

\section{References}

1. Møller CH, Penninga L, Wetterslev J, Steinbrüchel DA, Gluud C. Off-pump versus on-pump coronary artery bypass grafting for ischaemic heart disease. Cochrane Database Syst Rev 2012;CD007224.

2. van Dijk D, Spoor M, Hijman R, Nathoe HM, Borst C, Jansen EW, et al; Octopus Study Group. Cognitive and cardiac outcomes 5 years after off-pump vs on-pump coronary artery bypass graft surgery. JAMA. 2007;297:701-8.

3. Angelini GD, Taylor FC, Reeves BC, Ascione R. Early and midterm outcome after off-pump and on-pump surgery in Beating Heart Against Cardioplegic Arrest Studies (BHACAS 1 and 2): a pooled analysis of two randomised controlled trials. Lancet. 2002;359:1194-9.

4. Muneretto C, Bisleri G, Negri A, Manfredi J, Metra M, Nodari S, et al. Off-pump coronary artery bypass surgery technique for total arterial myocardial revascularization: a prospective randomized study. Ann Thorac Surg. 2003;76: 778-82; discussion 783.

5. Puskas JD, Williams WH, Mahoney EM, Huber PR, Block PC, Duke PG, et al. Off-pump vs conventional coronary artery bypass grafting: early and 1-year graft patency, cost, and quality-of-life outcomes: a randomized trial. JAMA. 2004;291: 1841-9.

6. Lee JD, Lee SJ, Tsushima WT, Yamauchi H, Lau WT, Popper J, et al. Benefits of off-pump bypass on neurologic and clinical morbidity: a prospective randomized trial. Ann Thorac Surg. 2003;76:18-25; discussion 25-6.

7. Karolak W, Hirsch G, Buth K, Légaré JF. Medium-term outcomes of coronary artery bypass graft surgery on pump versus off pump: results from a randomized controlled trial. Circulation. 2006;114(18 Suppl):363.

8. Widimsky P, Straka Z, Stros P, Jirasek K, Dvorak J, Votava J, et al. One-year coronary bypass graft patency: a randomized comparison between off-pump and on-pump surgery angiographic results of the PRAGUE-4 trial. Circulation. 2004; 110:3418-23.

9. Lingaas PS, Hol PK, Lundblad R, Rein KA, Mathisen L, Smith HJ, et al. Clinical and radiologic outcome of off-pump coronary surgery at 12 months follow-up: a prospective randomized trial. Ann Thorac Surg. 2006;81:2089-95.

10. Al-Ruzzeh S, George S, Bustami M, Wray J, Ilsley C, Athanasiou T, et al. Effect of off-pump coronary artery bypass surgery on clinical, angiographic, neurocognitive, and quality of life outcomes: randomised controlled trial. BMJ. 2006;332:1365.

11. Motallebzadeh R, Bland JM, Markus HS, Kaski JC, Jahangiri M. Healthrelated quality of life outcome after on-pump versus off-pump coronary artery bypass graft surgery: a prospective randomized study. Ann Thorac Surg. 2006; 82:615-9.

12. Mazzei V, Nasso G, Salamone G, Castorino F, Tommasini A, Anselmi A. Prospective randomized comparison of coronary bypass grafting with minimal extracorporeal circulation system (MECC) versus off-pump coronary surgery. Circulation. 2007;116:1761-7.

13. Hernandez F Jr, Brown JR, Likosky DS, Clough RA, Hess AL, Roth RM, et al. Neurocognitive outcomes of off-pump versus on-pump coronary artery bypass: a prospective randomized controlled trial. Ann Thorac Surg. 2007;84:1897-903.

14. Shroyer AL, Grover FL, Hattler B, Collins JF, McDonald GO, Kozora E, et al; Veterans Affairs Randomized On/Off Bypass (ROOBY) Study Group. On-pump versus off-pump coronary-artery bypass surgery. N Engl J Med. 2009;361: 1827-37.

15. Hueb W, Lopes NH, Pereira AC, Hueb AC, Soares PR, Favarato D, et al. Five-year follow-up of a randomized comparison between off-pump and on-pump stable multivessel coronary artery bypass grafting. The MASS III Trial. Circulation. 2010;122(11 Suppl):S48-52.

16. Fattouch K, Guccione F, Dioguardi P, Sampognaro R, Corrado E, Caruso M, et al. Off-pump versus on-pump myocardial revascularization in patients with ST-segment elevation myocardial infarction: a randomized trial. J Thorac Cardiovasc Surg. 2009; 137:650-6; discussion 656-7.

17. Sousa Uva M, Cavaco S, Oliveira AG, Matias F, Silva C, Mesquita A, et al. Early graft patency after off-pump and on-pump coronary bypass surgery: a prospective randomized study. Eur Heart J. 2010;31:2492-9. 
18. Møller CH, Perko MJ, Lund JT, Andersen LW, Kelbæk H, Madsen JK, et al. Three-year follow-up in a subset of high-risk patients randomly assigned to off-pump versus on-pump coronary artery bypass surgery: the Best Bypass Surgery trial. Heart. 2011;97:907-13

19. Takagi H, Yamamoto H, Iwata K, Goto SN, Umemoto T. Ask not which can impair early morbidity —ask which can improve late survival: a meta-analysis of randomized trials of off-pump versus on-pump coronary artery bypass. Int J Cardiol. 2012;158:435-8.

20. Angelini GD, Culliford L, Smith DK, Hamilton MC, Murphy GJ, Ascione R, et al. Effects of on- and off-pump coronary artery surgery on graft patency, survival, and health-related quality of life: long-term follow-up of 2 randomized controlled trials. J Thorac Cardiovasc Surg. 2009;137:295-303.

21. Karolak W, Hirsch G, Buth K, Légaré JF. Medium-term outcomes of coronary artery bypass graft surgery on pump versus off pump: results from a randomized controlled trial. Am Heart J. 2007;153:689-95.

22. Michaux I, Filipovic M, Skarvan K, Bolliger D, Schumann R, Bernet F, et al. A randomized comparison of right ventricular function after on-pump versus off-pump coronary artery bypass graft surgery. J Thorac Cardiovasc Surg. 2011;141:361-7.

23. Serrano CV Jr, Souza JA, Lopes NH, Fernandes JL, Nicolau JC, Blotta MH, et al. Reduced expression of systemic proinflammatory and myocardial biomarkers after off-pump versus on-pump coronary artery bypass surgery: a prospective randomized study. J Crit Care. 2010;25:305-12.

24. Puskas JD, Williams WH, O’Donnell R, Patterson RE, Sigman SR, Smith AS, et al. Off-pump and on-pump coronary artery bypass grafting are associated with similar graft patency, myocardial ischemia, and freedom from reintervention: long-term follow-up of a randomized trial. Ann Thorac Surg. 2011;91:1836-42; discussion 1842-3.
25. Zhang B, Zhou J, Li H, Liu Z, Chen A, Zhao Q. Comparison of graft patency between off-pump and on-pump coronary artery bypass grafting: an updated meta-analysis. Ann Thorac Surg. 2014;97:1335-41.

26. Takagi H, Watanabe T, Mizuno Y, Kawai N, Umemoto T. ALICE (All-Literature Investigation of Cardiovascular Evidence) Group. A meta-analysis of adjusted risk estimates for survival from observational studies of complete versus incomplete revascularization in patients with multivessel disease undergoing coronary artery bypass grafting. Interact Cardiovasc Thorac Surg. 2014;18:679-82.

27. Takagi H, Mizuno Y, Niwa M, Goto SN, Umemoto T. ALICE (All-Literature Investigation of Cardiovascular Evidence) Group. A meta-analysis of randomized trials for repeat revascularization following off-pump versus on-pump coronary artery bypass grafting. Interact Cardiovasc Thorac Surg. 2013; $17: 878-80$

28. Lamy A, Devereaux PJ, Prabhakaran D, Taggart DP, Hu S, Paolasso E, et al; CORONARY Investigators. Off-pump or on-pump coronary-artery bypass grafting at 30 days. $N$ Engl J Med. 2012;366:1489-97.

29. Devereaux PJ, Bhandari M, Clarke M, Montori VM, Cook DJ, Yusuf S, et al Need for expertise based randomised controlled trials. BMJ. 2005;330:88.

30. Lamy A, Devereaux PJ, Prabhakaran D, Taggart DP, Hu S, Paolasso E, et al CORONARY Investigators. Effects of off-pump and on-pump coronary-artery bypass grafting at 1 year. N Engl J Med. 2013;368:1179-88.

31. Diegeler A, Börgermann J, Kappert U, Breuer M, Böning A, Ursulescu A, et al; GOPCABE Study Group. Off-pump versus on-pump coronary-artery bypass grafting in elderly patients. N Engl J Med. 2013;368:1189-98.

32. Reeves BC, Deeks JJ, Higgins JP, Wells GA. Including non-randomized studies. In: Higgins JP, Green S, eds. Cochrane Handbook for Systematic Reviews of Interventions Version 5.1.0 (updated March 2011). The Cochrane Collaboration, 2011. Available at: http://www.cochrane-handbook.org. Accessed May 5, 2014

\title{
EDITORIAL COMMENTARY
}

\section{Should off-pump coronary artery bypass surgery be abandoned? A potential solution}

\author{
Harold L. Lazar, MD
}

See related article on pages 1820-9.

In this issue of the Journal, in a meta-analysis of 22 studies enrolling more than 100,000 patients, Takagi and coworkers ${ }^{1}$ conclude that coronary artery bypass grafting surgery (CABG) performed off-pump (OPCAB) is associated with worse long-term $(>5$ years) survival than on-pump CABG (ONCAB). ${ }^{1}$ The number of grafts performed per patient and the index of completeness of

\footnotetext{
From the Division of Cardiac Surgery, Boston Medical Center, Boston, Mass. Disclosures: Author has nothing to disclose with regard to commercial support. Address for reprints: Harold L. Lazar, MD, Division of Cardiac Surgery, Boston Medical Center, 88 E Newton St, Boston, MA 02118 (E-mail: harold.lazar@bmc.org). J Thorac Cardiovasc Surg 2014;148:1829-31 $0022-5223 / \$ 36.00$

Copyright (c) 2014 by The American Association for Thoracic Surgery http://dx.doi.org/10.1016/j.jtcvs.2014.10.002
}

revascularization were significantly greater for ONCAB than OPCAB patients. Furthermore, the need for repeat revascularization, recurrent angina, and rehospitalization for cardiac-related issues were also more frequent in the OPCAB group.

Previous studies have shown a $37 \%$ reduction in late mortality in patients undergoing CABG who have had a complete versus an incomplete revascularization. ${ }^{2}$ Several studies have reported a higher incidence of incomplete revascularization with OPCAB techniques, and this has been proposed as a mechanism for the decreased long-term survival in these patients. $^{2-7}$ OPCAB has also been associated with decreased graft patency, which may be responsible for decreased longterm survival and an increased need for repeated revascularization procedures. ${ }^{8}$ The results of the study by Takagi and coworkers $^{1}$ are similar to a recent Cochrane pooled analysis of data from more than 80 trials of ONCAB versus OPCAB that shows superior short-term and midterm survival with the ONCAB technique. 


\section{E-References}

E1. Brown JR, Hernandez F Jr, Klemperer JD, Clough RA, DiPierro FV, Hofmaster PA, et al. Long-term survival and cardiac troponin T elevation in on- and off-pump coronary artery bypass surgery. Heart Surg Forum. 2008; 11:E163-8.

E2. Bakaeen FG, Chu D, Kelly RF, Ward HB, Jessen ME, Chen GJ, et al. Performing coronary artery bypass grafting off-pump may compromise long-term survival in a veteran population. Ann Thorac Surg. 2013;95:1952-8; discussion 1959-60.

E3. Cooper WA, Thourani VH, Guyton RA, Kilgo P, Lattouf OM, Chen EP, et al. Racial disparity persists after on-pump and off-pump coronary artery bypass grafting. Circulation. 2009;120(11 Suppl):S59-64.

E4. Di Mauro M, Gagliardi M, Iacò AL, Contini M, Bivona A, Bosco P, et al. Does off-pump coronary surgery reduce postoperative acute renal failure? The importance of preoperative renal function. Ann Thorac Surg. 2007;84:1496-502.

E5. Filardo G, Grayburn PA, Hamilton C, Hebeler RF Jr, Cooksey WB, Hamman B. Comparing long-term survival between patients undergoing off-pump and on-pump coronary artery bypass graft operations. Ann Thorac Surg. 2011;92: 571-7; discussion 577-8.

E6. Fu SP, Zheng Z, Yuan X, Zhang SJ, Gao HW, Li Y, et al. Impact of off-pump techniques on sex differences in early and late outcomes after isolated coronary artery bypass grafts. Ann Thorac Surg. 2009;87:1090-6.

E7. García Fuster R, Paredes F, García Peláez A, Martín E, Cánovas S, Gil O, et al. Impact of increasing degrees of renal impairment on outcomes of coronary 2 artery bypass grafting: the off-pump advantage. Eur J Cardiothorac Surg. 2013;44:732-42.

E8. Gorki H, Patel NC, Panagopoulos G, Jennings J, Balacumaraswami L, Plestis K, et al. Off-pump coronary bypass surgery in patients with low ejection fraction: is there a long-term survival advantage? Innovations (Phila). 2010;5:33-41.
E9. Hu S, Zheng Z, Yuan X, Wang W, Song Y, Sun H, et al. Increasing long-term major vascular events and resource consumption in patients receiving off-pump coronary artery bypass: a single-center prospective observational study. Circulation. 2010;121:1800-8.

E10. Locker C, Schaff HV, Dearani JA, Daly RC. Improved late survival with arterial revascularization. Ann Cardiothorac Surg. 2013;2:467-74.

E11. Murzi M, Caputo M, Aresu G, Duggan S, Miceli A, Glauber M, et al. On-pump and off-pump coronary artery bypass grafting in patients with left main stem disease: a propensity score analysis. J Thorac Cardiovasc Surg. 2012;143:1382-8.

E12. Wu C, Camacho FT, Culliford AT, Gold JP, Wechsler AS, Higgins RS, et al. A comparison of long-term mortality for off-pump and on-pump coronary artery bypass graft surgery. Circ Cardiovasc Qual Outcomes. 2012;5:76-84.

E13. Raja SG, Husain M, Popescu FL, Chudasama D, Daley S, Amrani M. Does off-pump coronary artery bypass grafting negatively impact long-term survival and freedom from reintervention? Biomed Res Int. 2013;2013:602871.

E14. Robertson MW, Buth KJ, Stewart KM, Wood JR, Sullivan JA, Hirsch GM, et al. Complete revascularization is compromised in off-pump coronary artery bypass 3 grafting. J Thorac Cardiovasc Surg. 2013;145:992-8.

E15. Sarin EL, Kayatta MO, Kilgo P, Dara A, Puskas JD, Lattouf OM, et al. Short and long-term outcomes in octogenarian patients undergoing off-pump coronary artery bypass grafting compared with on-pump coronary artery bypass grafting. Innovations (Phila). 2011;6:110-5.

E16. Dalén M, Ivert T, Holzmann MJ, Sartipy U. Long-term survival after off-pump coronary artery bypass surgery: a Swedish nationwide cohort study. Ann Thorac Surg. 2013;96:2054-60.

E17. Synnergren MJ, Ekroth R, Odén A, Rexius H, Wiklund L. Incomplete revascularization reduces survival benefit of coronary artery bypass grafting: role of off-pump surgery. J Thorac Cardiovasc Surg. 2008;136:29-36. 